Instituto Internacional de Investigación y Desarrollo Tecnológico Educativo INDTEC, C.A.

DOI: https://doi.org/10.29394/Scientific.issn.2542-2987.2021.6.20.13.241-259

OAl-PMH: http://www.indteca.com/ojs/index.php/Revista Scientific/oai

Artículo Original / Original Article

\title{
Redefinición de las Artes como área de Aprendizaje en la Educación Primaria Venezolana
}

\author{
Autor: Yoslen Lisbardo Rodríguez \\ Universidad Fermín Toro, UFT \\ yoslen100@gmail.com \\ Barinas, Venezuela \\ https://orcid.org/0000-0003-3217-3136
}

Resumen

El objetivo de la presente investigación fue redefinir las artes como área de aprendizaje en la educación primaria venezolana. Se tomó como referencia las parroquias Corazón de Jesús. Alto Barinas y Ramón Ignacio Méndez del estado Barinas. La perspectiva metodológica utilizada fue a través de la interpretación que facilita la investigación cualitativa y la aplicabilidad del método hermenéutico. La técnica empleada fue la entrevista a profundidad apoyada en cuatros (4) informantes claves. La información aportada en las categorías se aplicó el proceso de triangulación. La teorización precisa que las artes están presentes en cada área de conocimientos existente, pero cómo una actividad complementaria. Las artes como área de conocimiento se definen como asignatura abarcarte de los campos que mueven los procesos creativos del ser. Con relación a la educación primaria en Venezuela, debe reconfigurarse el sistema a través de los procesos de formación y las nuevas prácticas educativas. El estudio condujo a redefinir las artes como un conocimiento holístico de todos los campos creativos cuyas expresiones pueden potenciarse en el desarrollo intelectual de los estudiantes de educación primaria, con trabajo interdisciplinar y colaborativo, y en prospectiva si se conjugan sus aprendizajes con los saberes artísticos- culturales de las bellas artes.

Palabras calve: artes; área; aprendizaje; educación primaria.

Código de clasificación internacional: 5802.03 - Desarrollo de asignaturas.

Cómo citar este artículo:

Rodríguez, Y. (2021). Redefinición de las Artes como área de Aprendizaje en la Educación

Primaria Venezolana. Revista Scientific, 6(20), 241-259, e-ISSN: 2542-2987. Recuperado de: https://doi.org/10.29394/Scientific.issn.2542-2987.2021.6.20.13.241-259

Fecha de Recepción: 13-01-2021
Fecha de Aceptación: 27-03-2021
Fecha de Publicación: 05-05-2021 
Instituto Internacional de Investigación y Desarrollo Tecnológico Educativo INDTEC, C.A.

DOI: https://doi.org/10.29394/Scientific.issn.2542-2987.2021.6.20.13.241-259

OAl-PMH: http://www.indteca.com/ojs/index.php/Revista Scientific/oai

Artículo Original / Original Article

\title{
Redefinition of the Arts as a Learning area in Venezuelan Primary Education
}

\begin{abstract}
The objective of this research was to redefine the arts as a learning area in Venezuelan primary education. The Heart of Jesus parishes were taken as a reference. Alto Barinas and Ramón Ignacio Méndez from the state of Barinas. The methodological perspective used was through the interpretation that facilitates qualitative research and the applicability of the hermeneutical method. The technique used was the in-depth interview supported by four (4) key informants. The information provided in the categories was applied the triangulation process. Theorization specifies that the arts are present in each area of existing knowledge, but as a complementary activity. The arts as an area of knowledge are defined as a subject encompassing the fields that move the creative processes of being. Regarding primary education in Venezuela, the system must be reconfigured through training processes and new educational practices. The study led to redefine the arts as a holistic knowledge of all creative fields whose expressions can be enhanced in the intellectual development of primary school students, with interdisciplinary and collaborative work, and prospectively if their learning is combined with artistic knowledgecultural fine arts.
\end{abstract}

Keywords: arts; area; learning; primary education.

International classification code: 5802.03 - Subject development.

How to cite this article:

Rodríguez, Y. (2021). Redefinition of the Arts as a Learning area in Venezuelan Primary Education. Revista Scientific, 6(20), 241-259, e-ISSN: 2542-2987. Recovered from: https://doi.org/10.29394/Scientific.issn.2542-2987.2021.6.20.13.241-259

Date Received: 13-01-2021
Date Acceptance: 27-03-2021
Date Publication: 05-05-2021 


\section{Introducción}

Las circunstancias que mueven al ser humano a ser parte de una acción en el mundo, traspasa los límites de la simple necesidad material por aquellas presente en su planos interno, busca expresarlo a través de las emociones, emociones que deben ser educadas indistintamente si los talentos son o no innatos. Todo espacio visual o auditivo en la sociedad está impregnado de los procesos creativos del ser, el cual constituye un arsenal de conocimiento que estudiado y trasmitido a profundidad se constituye en un campo epistemológico hacia las Artes como medio integral para la expresión de los hombres. El arte debe ser considerada desde cualquier contexto curricular como asignatura paralela para el desarrollo integral del hombre, orientadas desde principios que conlleven al desarrollo de estas.

El contexto escolar moldea elementos culturales presentes en los sistemas imperantes en cada país, es por ello, que la educación primaria es necesaria para permear la educación para el arte como asignatura de desarrollo integral, según la Organización de las Naciones Unidas para la Educación, la Ciencia y la Cultura (UNESCO, 2006a):

La educación en y a través de las artes también estimula el desarrollo cognitivo y hace que el modo y el contenido del aprendizaje resulte más pertinente para las necesidades de las sociedades modernas en las que vive el que lo recibe (pág. 2).

Entender el arte desde lo curricular como un hecho pedagógico unidisciplinario, es aislarlo del concepto de educación integral planteado para la educación entre las edades de seis y 12 años, etapa donde la capacidad de atención y práctica cognitiva se asocia a los procesos de expresión y creatividad involucrados en el desarrollo de las manifestaciones culturales de los pueblos.

En Venezuela la educación primaria ha pasado por un proceso de transformación curricular desde el año 1999 hasta nuestros días, donde los 
cambios se originan a partir de la construcción continua producto de las orientaciones anuales del Ministerio del Poder Popular para la Educación, sin embargo, luego de un proceso de revisión de los planes curriculares se evidencian hechos aislados de las artes como asignatura o área de aprendizaje, la mismas se verifica como actividades complementarias desde algunas áreas de conocimientos para la creación y producción artística transversalizado en algunos contenidos, sobre todo en el área de Lenguaje y Comunicación para atender las acciones comunicativas de expresión oral.

Resumiendo los aspectos del Currículo Nacional Bolivariano del Ministerio del Poder Popular para la Educación (2007a): "el lenguaje artístico como elemento de comunicación y expresión de la cultura y vida social [...] El lenguaje artístico para el desarrollo de la expresión del mundo exterior" (pág. 25); y atención al área de conocimiento Lenguaje, Comunicación y cultura y su vinculación con las artes, se contextualiza a partir del componente. El acercamiento hacia las artes en el plano curricular, se evidencia en las orientaciones pedagógicas en el año 2014-2015 con la formación de las grillas culturales que partía con el Plan de Masificación de las Artes y las Culturas, asimismo para el año 2016 la conformación de los grupos estables cuya finalidad era el accionar cultural y otros ámbitos de tipo socioeducativo. Entre otros aspectos que bordeaban las intencionalidades de las artes se menciona el Programa Nacional de Danza en el año 2002, y para el 2017 el movimiento teatral Cesar Rengifo, entre otros.

Cabe mencionar que las artes desde la educación primaria, es atendida a través de los componentes estructurales de la instituciones educativas y accionada por las coordinaciones de cultura, quienes tienen la función de articular pedagógicamente en la formación de los distintos campos como la música, artes plásticas, danza y teatro, entre otras, muchas de ellas son pinceladas que se debaten entre quienes tienen el perfil para dirigir y con algunos conocimientos de la totalidad de los campos, y quienes desconocen 
la universalidad de las artes, en tal sentido la interpretación de las acciones curriculares y los propósitos de los programas aún no se acercan a la concreción o proyección de educar integralmente.

La educación desde las artes viene a guiar la hipersensibilización de los talentos de los niños, niñas y adolescentes, y a contribuir con la cultura general de una sociedad, no solo se aprenden las áreas de conocimientos primordiales en los primeros años de estudio, porque estaríamos apostando a aprendizajes conductistas. La trascendencia del conocimiento conjuga la enseñanzaaprendizaje transdisciplinaria con las particularidades y las complejidades del ser, como un hecho semántico, creativo, etéreo y proyectivo que pudiese ser cuestionable, modificable, ampliado, cíclico, entre otros principios de la red compleja del saber de las artes que se asume también por la cientificidad.

En este contexto, interpretando a Ivars y Wight (2010): el arte se constituye en un sistema metaestable, en otras palabras, se abre a la posibilidad de seguir tejiendo el conocimiento como lo hace la ciencia y la filosofía, no niega el acceso a las posibilidades de experiencias distintas desde la creatividad del ser, ni a la producción humana que viene marcada de manera compleja por un proceso evolutivo y autoorganizativo, en consecuencia, los sistemas deben estar abiertos a la compresión paradigmática dentro y fuera de las artes, para la multiplicidad de la producción artística como fuente generadora del ser.

En función a lo anteriormente expuesto, se describe la realidad del subsistema de educación primaria en Venezuela donde a través de la revisión de orientaciones educativas entre los años 2002 hasta el presente así como los planes, programas y proyectos del Ministerio del Poder Popular para la Educación (MPPE), se evidencia que las artes están siendo permeadas desde la práctica docente como un hecho de aprendizaje aislado, de completud de un programa y disgregado en varias áreas de conocimiento y no como una asignatura integral asumida como las demás áreas de aprendizajes en el plano 
curricular y accionar pedagógico.

De esta realidad no escapan las escuelas del estado Barinas, Venezuela, específicamente las que integran las parroquias Corazón de Jesús, Parroquia Alto Barinas, y Ramón Ignacio Méndez, donde se observa a través de la revisión de actividades de las Coordinaciones de Cultura de algunas instituciones, que las artes que integran los campos de la música, la danza, las artes escénicas, entre otras, no se constituyen como una área de aprendizaje, sino como componente complementario que transversaliza las demás áreas de conocimiento.

En este contexto el propósito de este artículo es redefinir las artes como área de aprendizaje en la Educación Primaria Venezolana desde una perspectiva compleja. La reorganización desde el esquema complejo resignificaría las aristas que encierran estos campos para unificar criterios de los procesos creativos de las artes y la pedagogía como medio que encierra la interdisciplinaridad asumida desde el contexto educativo que representa la educación primaria en Venezuela.

\section{Referentes Teóricos}

\subsection{Antecedentes de la investigación}

La revisión de algunas investigaciones relacionadas con el presente estudio se menciona a Ríos (2018): cuyo trabajo estuvo orientado a la formación en artes visuales a la práctica educativa a través de las narrativas en torno a la constitución de identidades profesionales y tránsitos vividos por docentes noveles. El propósito fue esbozar las inquietudes de los docentes que se integran en el campo educativo para llevar a cabo las responsabilidades de las artes y sus procesos de transferibilidad, la autobiografía caracteriza las particularidades de quienes están contenidos como artistas desde las experiencias y las formas de exteriorizarlo es un ejemplo para los diversos campos de las artes. 
Las terminaciones de este estudio llevaron a reflexionar en que hay nuevas formas de investigar y constituir conocimiento, que la formación de nuevos conocimientos fortalece la identidad tanto como docente y artista. Asimismo, reflexionó el valor de elementos del contexto que no tienen la misma importancia para todos. A la postura de un docente formado para las artes en función de la construcción continua de una sociedad algo compleja pero que necesita interiorizarse siendo las artes visuales una manera de proyectarlo.

Entre otras de las investigaciones consultadas, se menciona a Martínez (2017): donde destaca la importancia de las artes como mediador de los aprendizajes en las etapas escolares infantiles, primaria, secundaria y bachillerato. La relevancia del estudio de este autor, busca contribuir con la optimización de la educación a través de la gestión de las Artes como elemento generador de la creatividad, la cultura, la interdisciplinariedad y la experimentación transversalizando los aprendizajes con las otras áreas de conocimiento tomando dichas sugerencias como asignatura formal del currículo.

Entre las reflexiones finales de la investigación se describe que el desarrollo de proyectos de arte y cultura en los centros educativos utilizando el arte; fomenta el viaje multidisciplinar (científico-artístico-cultural) constituyéndose como una herramienta idónea desde el currículo para atender desde la etapa infantil hasta bachillerato. Considerada como una propuesta se busca a futuro implementarlas e irla adaptando producto de los distintos proyectos artísticos intrínseco en las artes.

\subsection{Las artes como área de aprendizaje}

Las artes constituyen el medio de expresión del hombre a través de diversos lenguajes, evocando las ideas internas y proyectándolas al mundo de forma creativa. El arte tiene su origen en la prehistoria, cuando en las cavernas según la historia mostraban sus experiencias, las necesidades a través 
narrativas en forma de imágenes, también llamadas pinturas rupestres, cabe destacar que la necesidad imperiosa del hombre por expresar los que sabe y tiene en su estructura mental y emocional es vital para la reconstrucción de los planos internos y externos del ser. Considerando la idea anterior sobre el arte, Arnheim (1993), amplia que es:

Uno de los instrumentos más poderosos de que disponemos para la realización de la vida. Percibir en toda su plenitud, amar, interesarse por algo, comprender, crear, descubrir, anhelar o esperar son las acciones de toda actividad artística y, en sí mismo, el valor supremo de la vida. El arte es un medio fundamental de orientación, nacido de la necesidad que la persona tiene de comprenderse a sí mismo y al mundo en el que habita (pág. 35).

Existes diversos campos que permiten al ser expresar esos lenguajes creativos, entre ellos según la hoja de ruta de la Organización de las Naciones Unidas para la Educación, la Ciencia y la Cultura (UNESCO, 2006b): menciona "[...] las artes escénicas (danza, teatro, música, etc.), la literatura y la poesía, la artesanía, el diseño, el arte digital, la narración, el patrimonio, las artes visuales y, finalmente, el cine, la fotografía y los medios" (pág. 5). Aunque existen un campo muy diverso las mismas se reconstituyen de acuerdo con los espacios y territorios nutridos por la cultura que envuelve esas sociedades, conocimiento que debe ser reconocido e insertado académicamente en los currículos respectivos como área de conocimiento o aprendizaje, y allí aplicar las taxonomías correspondientes a las competencias de los grados y campos respectivos.

Generar la atención sobres las cualidades creativas del ser es de importancia, porque al encontrar mecanismos que regulen el potencial intelectual de expresión se desarrolla en cada ser humano el sentido de pertenencia con el mundo que lo rodea, y las producciones son aportes de crecimiento a una sociedad que está permeando en cada acción con el hecho 
educativo.

El arte no es exclusiva de los que poseen talentos para sus campos específicos, es para comprensión y expresión de todos, de allí lo que se considera cultura general, cuando ese todo es la simplicidad de cada saber, de cada conocer, momento donde la reflexión en el enfoque de la complejidad toma ventajas para hablar de educación desde el desacierto y a la redefinición de educar para el reencuentro del ser y para luego enfocarse en el mundo material.

Existe una serie de investigadores que dan forma y consistencia a las ideas de las artes como asignatura en los distintos pensum de estudios, entre ellos; los que proyecta la creatividad en primer orden, se mencionan los más resaltantes a Pestalozzi (1746-1827), cuyo conocimiento del dibujo y la música apostaba a la democratización del saber y sentido estético a través de la educación artística. De esta manera, se presenta el análisis de Froebel (17821852), en la Universalidad de lo estético y en desarrollo de las potencialidades de los niños. Contextualizando los estudios de Herbert Read (1893-1968), las artes fomentan un aptitud y actitud acertada, sensible, optimista, colectiva a través de la formación, por lo tanto, busca el mismo objetivo que la educación.

Siguiendo el apartado anterior, se adicionan estudios valorativos como el de Viktor Lowenfeld (1903-1960), y las cuatro etapas de desarrollo creativo (garabateo, preesquemática, realismo y pseudonaturalismo) todos ellos adquiridos desde los dos años hasta los 14 años. En este orden de ideas, Gardner (2000), citado por Macías (2002): menciona que "los seres humanos pueden conocer el mundo de ocho modos diferentes, [...] las ocho inteligencias humanas: lingüística, lógico-matemática, espacial, musical, corporal, naturalista, interpersonal e intrapersonal" (pág. 35). Sin embargo, en el plano individual educativo la práctica de todas llevaría al reconocimiento de los talentos en los primeros años de vida.

Los estudios y enfoques, y profundización de los mismos en materia de 
las artes, busca la aproximación de la articulación educativa con las emociones y expresiones del ser producto del proceso creativo que ocurre internamente, por ende la enseñanza y aprendizajes de las artes como una asignatura es necesaria para darle forma, definición y reorganización al conocimiento de la música, el teatro, la literatura, la pintura, la poesía, artes visuales, así como otros conocimientos emergentes, que encierran las artes pero ya en sentido estricto y obligatorio para el contexto curricular y pedagógico.

\subsection{La educación primaria en Venezuela}

La educación primaria constituye el nivel de estudio que corresponde a las edades de aprendizaje entre los seis y 12 años, etapa donde se desarrollan distintas actitudes y aptitudes que les permiten a los niños el desarrollo integral con miras a la adolescencia. La etapa primaria forma parte de una estructura o sistema territorializada por características de una sociedad, no obstante, establece una relación multidisciplinar con otros sistemas macros que corresponden a beneficios y derecho educativos que atañen a política educativas internacionales que hacen la educación y los aprendizajes un hecho colaborativo sin fronteras.

En Venezuela, la educación primaria se constituye en un subsistema y forma parte de un sistema educativo nacional bajo directrices sociopolíticas que emergen de los planes de la nación, se rige pedagógicamente por un currículo o plan de trabajo disgregado por grados, los cuales están esquematizados por componentes según la etapa en áreas de conocimiento, que a su vez presenta unas finalidades por área con sus respectivos contenidos. El Ministerio del Poder Popular para la Educación (2007b), define que el subsistema de Educación Primaria, tiene por finalidad:

[...] Formar niños y niñas activos, reflexivos, críticos e independientes, con elevado interés por la actividad científica, humanista y artística; con un desarrollo de la comprensión, confrontación y verificación de su realidad por sí mismos y sí 
mismas; con una conciencia que les permita aprender desde el entorno y ser cada vez más participativos, protagónicos y corresponsables en su actuación en la escuela, familia y comunidad (pág. 12).

La educación primaria para verificar estas finalidades presentadas, busca en los docentes y demás protagonistas del proceder pedagógico la materialización de los contenidos curriculares en los Proyecto Educativo Integral Comunitario (PEIC) institucionales y Proyectos de Aprendizajes (PA), cada escuela es una realidad distinta, pero las mismas confluyen en los planes, programas y proyectos emanados por el Ministerio del Poder Popular para la Educación y las orientaciones respectivas. Cabe mencionar que los proyectos de aprendizaje son el pilar fundamental del conocimiento que van a recibir y experimentar los estudiantes, están transversalizados por diferentes ejes integradores y articulados de alguna forma con las intencionalidades permeadas en el currículo.

La eficacia y calidad de la educación primaria en Venezuela depende de la gestión político educativo en cuanto a la formación docente, implementación de recursos tecnológicos (docentes-estudiantes), instalaciones dotadas (servicio de alimentación, aulas acondicionadas, recursos materiales) y de programas continuos que coordinen lo social y cultural como un hecho de transformación de una sociedad, en consecuencia, el cumplimiento y evaluación de lo aspirado por el sistema educativo se vuelve tema de discusión frente al impacto de los resultados en la implementación tanto curricular así como del accionar pedagógico.

\section{Metodología}

El transitar ontológico de la realidad estudiada se esboza desde el paradigma cualitativo, porque describe las realidades suscitadas en un contexto, en tal sentido, la descripción y la interpretación esquematiza los 
fenómenos de estudio que enmarcan la investigación y sus procesos, momento para que las relaciones intersubjetivas, den forma a las categorías de estudio.

La investigación se describe como cualitativa, porque señalando a Trujillo, Toro, Lomas y Merlo (2019): "se fundamenta en las ideas del paradigma interpretativista, desarrollado por las Ciencias Sociales, según el cual, no existe una realidad social única, más bien, variadas realidades construidas desde la óptica personal de cada uno de los individuos" (págs. 2223). En consecuencia, la interpretación de los hechos subjetivos de personas o grupos queda sometida a las formas de observar, interpretar y describir al sujeto-objeto estudiado.

Igualmente, se asume desde el enfoque complejo, porque trascienda la tradicionalidad de un hecho concreto y pluraliza las particularidades y rupturas, acuerdos y desacuerdos, mostrando la realidad tal como sucede. Se busca reorganizar metodológicamente los constructos teóricos hacia nuevas redefiniciones que delinean las artes como área de aprendizaje en la educación primaria venezolana. El comprender la educación como un hecho parcelado, y aun pensando en la integralidad de esta, se conjuga la necesidad de avizorar nuevas ideas que están presente y deben ser expuestas.

La actividad interpretativa de la investigación se accionó a partir del método hermenéutico en circunstancias encontradas, revisión de textos, posturas discursivas y documentos que conllevaron los cánones primarios del estudio para dirigirlo a las teorizaciones formales de las interpretaciones. Adicionalmente Arteta (2017): manifiesta que "la hermenéutica tiene como método la fenomenología porque entiende el comprender como un fenómeno cuyo propósito es encontrar el sentido que tiene el texto. Es una ciencia de límites porque hasta dónde llega el lenguaje llega la comprensión” (pág. 17).

El estudio requirió de informantes claves o potenciales, para darle paso a esa intersubjetividad de los actores, el proceso de selección de los 
informantes está relacionado con la interacción o acercamiento del investigador con personas relacionadas y con la experiencias de las categorías estudiadas, para ellos se contó con cuatro informantes clave, dos docentes de danza y con experiencia como coordinadores del área de cultura, un docente de aula conocedor de aspectos relacionados al arte, y un artista-docente.

El proceso de recolección de información se llevó a cabo a través de un guion de entrevista vinculado con las categorías de estudio. La entrevista a profundidad permitió de forma progresiva incorporar las ideas de los informantes claves. Para Schettini y Cortazzo (2016): la entrevista a profundidad busca "[...] comprender las perspectivas y los significados que los informantes tienen sobre sus vidas y sus experiencias, expresándolas con sus propias palabras, por lo que las entrevistas en profundidad [...]" (pág. 21).

La técnica de análisis de la información aplicada fue la triangulación, respaldada por cada una de las fuentes informativas, y contrastadas siguiendo la ilación de las categorías. La triangulación, en conformidad con Alzás y Casa (2017): "es considerada como un mecanismo para evitar el sesgo que provoca la persona que investiga" (pág. 415); en tal sentido, la interpretación de las unidades de análisis, son comprendidas para reconfigurar la teorización expuestas en cada una de las categorías.

A continuación, en el cuadro 1, se exponen algunos extractos relacionados a las categorías aportados por los informantes:

Cuadro 1. Matriz de categorización.

\begin{tabular}{|c|c|c|c|c|c|}
\hline \multirow[t]{2}{*}{ Categorías } & $\begin{array}{c}\text { Informantes } \\
\text { Clave }\end{array}$ & Informante & Informante & Informante & Informante \\
\hline & Subcategorías & 1 & 2 & 3 & 4 \\
\hline $\begin{array}{l}\text { Las artes } \\
\text { como área de } \\
\text { aprendizaje }\end{array}$ & $\begin{array}{c}\text { Consideraciones } \\
\text { Conceptuales } \\
\text { de las artes }\end{array}$ & $\begin{array}{c}\text { Son } \\
\text { emociones } \\
\text { emanadas } \\
\text { del alma y } \\
\text { que el cuerpo } \\
\text { quiere } \\
\text { expresar de } \\
\text { alguna } \\
\text { forma... }\end{array}$ & $\begin{array}{l}\text { Considero que } \\
\text { están en todo } \\
\text { lo que } \\
\text { observamos y } \\
\text { las forma de } \\
\text { describir lo que } \\
\text { procesamos } \\
\text { desde el } \\
\text { conocimiento y } \\
\text { las } \\
\text { sensaciones }\end{array}$ & $\begin{array}{c}\text { Construimos cada } \\
\text { día un pedazo para } \\
\text { el arte, redefinimos } \\
\text { una y otra vez lo } \\
\text { que sentimos, y a } \\
\text { su vez } \\
\text { compartimos con el } \\
\text { exterior, es decir } \\
\text { ese universo } \\
\text { cargado de } \\
\text { conocimiento }\end{array}$ & $\begin{array}{c}\text { Están sujetas a } \\
\text { lo que pintamos, } \\
\text { cantamos, } \\
\text { escenificamos } \\
\text { hasta lo que } \\
\text { expresamos de } \\
\text { forma escrita. } \\
\text { No se limita a } \\
\text { una persona } \\
\text { con aptitudes de } \\
\text { artísticas, sino }\end{array}$ \\
\hline
\end{tabular}


Instituto Internacional de Investigación y Desarrollo Tecnológico Educativo INDTEC, C.A.

DOI: https://doi.org/10.29394/Scientific.issn.2542-2987.2021.6.20.13.241-259

OAI-PMH: http://www.indteca.com/ojs/index.php/Revista Scientific/oai

Artículo Original / Original Article

\begin{tabular}{|c|c|c|c|c|c|}
\hline & & & $\begin{array}{c}\text { internas se } \\
\text { proyecta al } \\
\text { mundo... }\end{array}$ & $\begin{array}{c}\text { etéreo, porque } \\
\text { antes de saber, } \\
\text { primero sentimos } \\
\text { ¡eso es el arte! }\end{array}$ & $\begin{array}{c}\text { que todos } \\
\text { debemos } \\
\text { experimentar las } \\
\text { artes... } \\
\end{array}$ \\
\hline & $\begin{array}{l}\text { Enseñanza de } \\
\text { las Artes }\end{array}$ & $\begin{array}{l}\text { Las artes } \\
\text { siempre han } \\
\text { estado allí } \\
\text { para contar, } \\
\text { calcular, } \\
\text { crear, } \\
\text { dialogar, pero } \\
\text { que los } \\
\text { pensum de } \\
\text { estudios no la } \\
\text { incluyan } \\
\text { como } \\
\text { asignatura } \\
\text { entonces } \\
\text { están aún } \\
\text { trabajando } \\
\text { parcelado... }\end{array}$ & $\begin{array}{c}\text { Desde el arte } \\
\text { se guía al } \\
\text { estudiante a } \\
\text { profundizar y a } \\
\text { reflexionar } \\
\text { hacia otras } \\
\text { asignaturas. } \\
\text { Repensarla } \\
\text { como un área } \\
\text { del saber, que } \\
\text { sería un } \\
\text { enfoque } \\
\text { integral para } \\
\text { que el ser } \\
\text { humano se } \\
\text { sensibilice } \\
\text { más... }\end{array}$ & $\begin{array}{l}\text { Si seguimos el } \\
\text { proceso de } \\
\text { articulación es } \\
\text { bueno, pero es } \\
\text { mejor profundizarla } \\
\text { como un área de } \\
\text { conocimiento y } \\
\text { desde allí articular } \\
\text { con otras aéreas... }\end{array}$ & $\begin{array}{c}\text { Más allá de } \\
\text { globalizar las } \\
\text { artes, es más } \\
\text { asertivo enseñar } \\
\text { a todos con los } \\
\text { talentos y con el } \\
\text { descubrimiento } \\
\text { de los talentos y } \\
\text { ese sería el } \\
\text { punto de partida } \\
\text { para explotar la } \\
\text { creatividad en } \\
\text { otras áreas... }\end{array}$ \\
\hline $\begin{array}{l}\text { Educación } \\
\text { Primaria en } \\
\text { Venezuela }\end{array}$ & $\begin{array}{c}\text { Permeabilidad } \\
\text { de los procesos } \\
\text { de enseñanza }\end{array}$ & $\begin{array}{c}\text { El enseñar } \\
\text { por proyecto } \\
\text { es importante } \\
\text { pero la } \\
\text { preparación } \\
\text { del maestro } \\
\text { es más aún... }\end{array}$ & $\begin{array}{c}\text { Garantizar el } \\
\text { aprendizaje de } \\
\text { primaria cobra } \\
\text { vida cuando el } \\
\text { aseguramiento } \\
\text { de los } \\
\text { programas es } \\
\text { de calidad... }\end{array}$ & $\begin{array}{l}\text { La educación en } \\
\text { Venezuela debe } \\
\text { retomar la } \\
\text { interdisciplinariedad } \\
\text { en la práctica } \\
\text { educativa... }\end{array}$ & $\begin{array}{c}\text { La educación } \\
\text { primaria debe } \\
\text { afianzarse con } \\
\text { valores } \\
\text { culturales y } \\
\text { sociales en } \\
\text { correspondencia } \\
\text { con la } \\
\text { realidad... }\end{array}$ \\
\hline
\end{tabular}

Fuente: El Autor (2021).

\section{Resultados}

Una vez realizados los procesos de categorización y triangulación de la información se procedió a evocar de manera precisa las interpretaciones generadas de los informantes claves, evitando cualquier sesgo o respuesta que llevaran a otro lugar las categorías de estudio.

Con respecto a las implicaciones de las Artes como área de conocimiento; se precisó que las mismas están presentes en cada área de conocimiento existente en el currículo actual, y estrategia de articulación de los aprendizajes como un medio de expresión, sin embargo, profundizando a través de las artes y sus campos ayudaría a los niños a interiorizar la musicalidad, la pintura, las esculturas, las escenas, la narrativa, artes visuales entre otras.

En el mismo orden de ideas, la búsqueda incesante del ser por la 
creatividad es más fácil si se trabaja el descubrimiento de los talentos, razón por la cual las artes se consolidan con otros conocimientos que ayudan en los procesos cognitivos. Estos procesos cognitivos relacionan las áreas de aprendizaje presentes en una estructura curricular y son articuladas con otras denominadas complementarias. En este contexto las artes como área de aprendizaje de un currículo se define como asignatura abarcarte de los campos que mueven los procesos creativos del ser, desde un enfoque complejo y dialógico como lo hacen otras áreas de aprendizajes, poniendo en práctica la interdisciplinariedad y la didáctica de articulación de los aprendizajes inmersos en los proyectos.

Con relación a la educación primaria en Venezuela, apuntala hacia el deber ser y la configuración del sistema a través de los procesos de formación, el aseguramiento de la interdisciplinariedad, y nuevas prácticas educativas afianzando en los niños valores sociales, culturales adaptados a la realidad del país. Confrontar el conocimiento con la cientificidad y la sensibilidad social para mantener activas directrices educativas que buscan la responsabilidad, cooperación, construcción colectiva, participación, entre otros. La preparación en educación primaria debe potenciar el arsenal humano que posee cada niño, debe perfilarse con las competencias y al mismo tiempo fomentar valores de sensibilidad por el contexto geográfico donde se desenvuelve.

Las artes como área de aprendizaje en educación primaria se reconfigura en el conocimiento emergente de los campos creativos del ser, de enfoque cooperativo, colaborativo, transdisciplinar y complejo, en el desarrollo cognitivo de cada niño y niña con potencialidades artísticas o no, cuyo propósito es el descubriendo de nuevos conocimientos y en trascendencias de otros presentes en la intencionalidades curriculares en la educación primaria venezolana, mejorando la cientificidad y sensibilidad social de cada estudiante por su contexto geográfico el cual proyectará por medio de los campos artístico y dejando huellas imborrables e inéditas como aportes a la educación quien lo 
cobijó en una etapa de aprendizaje de sus vidas.

\section{Conclusiones}

La búsqueda de conocimiento del ser humano ha estado supeditada casi siempre por cánones de enseñanza que buscan a darle respuesta a cada pregunta que se haga en base a formulas procesos, análisis entre otras estrategias curriculares dotada de rigidez, es por ello, que surge la necesidad imperiosa de darle perspectiva a aspiraciones que consoliden las artes como área de aprendizaje en la educación primaria, en este caso se delimitó a Venezuela.

El proceso de revisión literaria, documentos y otras fuentes para la consolidación teórica sustentaron las categorías de estudio, definiendo las artes como medios de expresión del hombre para describir sus emociones en atención a los procesos creativos presentes en los diversos campos como: las artes musicales, escénicas, literarias, visuales, digitales, entre otras que se siguen generando en la actualidad. Las mismas se han visto como exclusivas de quienes poseen esos talentos y potencialidades artísticas. Hecho que desde la educación primaria es comprendido a través de los procesos de articulación de los aprendizajes presente en los proyectos institucionales concatenados al Currículo Nacional Bolivariano.

El encuentro interpretativo de las categorías de estudio, se vinculó al proceder metodológico cuando se asumió la investigación cualitativa como medio para describir e interpretar las realidades del contexto. El método hermenéutico permitió el equilibrio ontológico de la investigación, cuando posterior a la revisión teórica, los informantes claves aportaron ideas que se configuraron de forma intersubjetiva para visualizar las categorías que fueron contrastadas a través del proceso de triangulación con una visión hacia la redefinición teórica.

La reflexión sobre las artes como área de aprendizaje en la educación 
primaria venezolana se redefine como: la cognición holística de las expresiones del ser por medio de los diversos campos creativos de las artes, potenciando el desarrollo intelectual de los estudiantes de educación primaria, sumergidos en una relación interdisciplinaria y colaborativa, creando una etapa de conocimiento, que en prospectiva afianzaría la cientificidad como futuro profesional que conjugó sus aprendizaje con los saberes artísticos y la cultura general de las bellas artes.

La obligatoriedad y pertenencia curricular para las artes, dependerá de la aceptación de los sistemas educativos a reconsiderar los componentes con fines más humanistas, artísticos y filosóficos, en una sociedad que hace, habla y escribe de ciencias, y ellas está irremediablemente la sensibilidad del hombre en la reconstrucción continua de la sociedad.

\section{Referencias}

Alzás, T. \& Casa, L. (2017). La evolución del concepto de triangulación en la investigación social. Revista Pesquisa Qualitativa, 5(8), 395-418, eISSN: 2525-8222. Recuperado de:

https://editora.sepq.org.br/rpq/article/view/95

Arnheim, R. (1993). Consideraciones sobre la educación artística. ISBN: 978-84-750-9877-7. Barcelona, España: Ediciones Paidós Ibérica, S.A. Arteta, C. (2017). Hermenéutica, Pedagogía y Praxeología. ISBN: 978-9589145-62-3. Barranquilla, Colombia: Universidad Libre Seccional Barranquilla. Recuperado de: https://doi.org/10.18041/978-958-9145$\underline{69-2}$

Ivars, J., \& Wight, G. (2010). Sobre la Complejidad II. Zehar: revista de Arteleku-ko aldizkaria, (67), 1-48, e-ISSN: 1133-844X. Recuperado de: http://artxibo.arteleku.net/es/islandora/object/arteleku\%3A6427

Macías. M. (2002). Las Múltiples Inteligencias. Psicología desde el Caribe, (10), 27-38, e-ISSN: 0123-417X. Recuperado de: 
https://www.redalyc.org/articulo.oa?id=21301003

Martínez, M. (2017). El uso del arte como mediador del aprendizaje en la etapa escolar (Infantil, Primaria, Secundaria y Bachillerato). Tesis

Doctoral. Madrid, España: Universidad Complutense de Madrid.

Recuperado de: https://eprints.ucm.es/id/eprint/48662/1/T40416.pdf

Ministerio del Poder Popular para la Educación (2007a,b). Currículo del

Subsistema de Educación Primaria Bolivariana. ISBN: 978-980-218-

283-1. Caracas, Venezuela: Fundación Centro Nacional para el Mejoramiento de la Enseñanza de la Ciencia - CENAMEC.

Ríos, M. (2018). De la formación en artes visuales a la práctica educativa: Narrativas en torno a la constitución de identidades profesionales y tránsitos vividos por docentes noveles. Tesis Doctoral. Barcelona, España: Universitat de Barcelona. Recuperado de:

http://hdl.handle.net/10803/587225

Schettini, P., \& Cortazzo, I. (coords.) (2016). Técnicas y Estrategias en la Investigación Cualitativa. Primera edición, ISBN: 978-950-34-1327-2.

Buenos Aires, Argentina: Universidad Nacional de La Plata.

Trujillo, C., Toro, M., Lomas, K., \& Merlo, M. (2019). Investigación Cualitativa: Epistemología, Consentimiento Informado, Entrevista en Profundidad. Primera, edición, ISBN: 978-9942-35-555-3. Ibarra, Ecuador: Universidad Técnica del Norte.

UNESCO $(2006 a, b)$. Hoja de Ruta para la Educación Artística. Lisboa, Portugal: Conferencia Mundial sobre la Educación Artística: construir capacidades creativas para el siglo XXI. 


\section{Yoslen Lisbardo Rodríguez \\ e-mail: yoslen100@gmail.com}

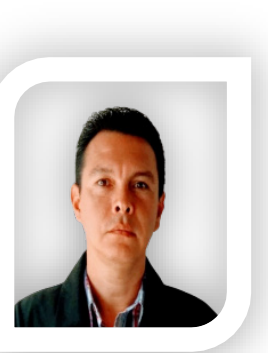

Nacido en el estado Barinas, Venezuela, el 7 de mayo del año 1974. Actualmente me desempeño como docente de aula en la Escuela Básica Bolivariana "Ciudad Varyná", del estado Barinas, Venezuela; mis estudios académicos se basan en la Especialización en Educación Comunitaria por la Universidad Pedagógica Experimental Libertador (UPEL), Barinas; Profesor en Educación Integral de la Universidad Pedagógica Experimental Libertador - Instituto de Mejoramiento Profesional del Magisterio (UPEL-IMPM), Barinas, Venezuela; Experiencias como tutor en pregrado y asesor en trabajos de investigación a nivel de especialización y maestría; he participado como Ponente en el Congreso Internacional de Investigación "Innovación y Buenas Prácticas en Educación" de la Universidad Pedagógica Experimental Libertador (UPEL) y la Universidad Nacional Abierta (UNA) en diciembre del año 2019.

El contenido de este manuscrito se difunde bajo una Licencia de Creative Commons ReconocimientoNoComercial-Compartirlgual 4.0 Internacional 